\title{
The Analytical Transmission Electron Microscopy: A Powerful Tool for the Investigation of Low-Dimensional Carbon Nanomaterials
}

\author{
Stefano Casciardi, ${ }^{1}$ Renata Sisto, ${ }^{1}$ and Marco Diociaiuti ${ }^{2}$ \\ ${ }^{1}$ Department of Occupational Hygiene, National Institution for Insurance against Accidents at Work (INAIL Research), \\ Monte Porzio Catone, Rome, Italy \\ ${ }^{2}$ Department of Technologies and Health, Italian National Institute of Health (ISS), Rome, Italy
}

Correspondence should be addressed to Stefano Casciardi; s.casciardi@inail.it

Received 15 April 2013; Accepted 14 October 2013

Academic Editor: Nadya Mason

Copyright (C) 2013 Stefano Casciardi et al. This is an open access article distributed under the Creative Commons Attribution License, which permits unrestricted use, distribution, and reproduction in any medium, provided the original work is properly cited.

\begin{abstract}
Chemical and physical characterization of nanomaterials is essential to improve synthesis processes, for new technological and commercial applications, and to assess their toxicity through in vitro and in vivo studies. New nanomaterials and new synthesis processes are continuously tested and updated to exploit their innovative properties. In this paper, low-dimensional carbon nanostructure characterization was performed using analytical transmission electron microscopy. Conventional and advanced microscopy techniques, such as acquisition of high resolution images, nanobeam electron diffraction patterns, X-ray energy dispersion, and electron energy loss spectra, were used to determine the main physical and chemical properties of single wall and multiwall carbon nanotubes, graphene flakes, and amorphous carbon films. Through the resulting micrographs, diffraction patterns, and spectra, the main low-dimensional carbon nanostructures properties were determined in terms of structural defects and/or the presence of metallic or heavy elements, such as those used as catalyst or to decorate nanotubes. The obtained information is of crucial importance to investigate low-dimension nanomaterial biological activity.
\end{abstract}

\section{Introduction}

Among low-dimensional carbon nanostructures, nanotubes play the main role up to now. They were discovered by chance in 1991 by the physicist Sumio Iijima (NEC Corporation, Tsukuba, Ibaraki, Japan), in the analysis of the products obtained in the growth of fullerenes, the third allotropic species of carbon [1]. Actually, in the late 1950s, researchers, Roger Bacon (Union Carbide Corporation, Cleveland, $\mathrm{OH}$, USA) and then in the 1970s and 1980s (Morinobu Endo), had unknowingly observed nanotubes but had not recognized them as such $[2,3]$.

As for all materials of nanometric dimension, nanotubes have technical characteristics making them especially attractive in a wide range of applications [4-16]. There are numerous sectors in which the specific properties of carbon nanotubes can be used, and there are many other potential sectors where applied research is investing considerable resources. For example, these materials are very resistant to traction. Indeed, it can be said that carbon nanotubes, without structural defects, are the most resistant organic material. Nanotubes are likewise very light and highly flexible and can be folded repeatedly up to $90^{\circ}$ without being damaged. All these properties make them the best materials available today for reinforcing fibres in high performance composite materials, replacing and having definitely superior properties than the natural or artificial fibres used up to now, for example, carbon fibres, Kevlar, or glass wool, to mention but a few.

Although the basic element of carbon nanotubes is the graphitic sheet, the good electrical conducting properties of graphite appear only partially in nanotubes, which are electronic hybrids. This fact suggests the use of nanotubes for manufacturing much smaller electronic devices and with much higher performance levels compared to the ones that are currently based on silicon physics. Carbon nanotubes can 
be used to make diodes, transistors, LEDs, ultraviolet lasers, photovoltaic cells, electronic cannons for producing ultrahigh-definition plasma screens, and much more $[17,18]$.

Another property of nanotubes is their capillarity due to the tubular shape and high surface area/weight ratio. This characteristic makes nanotubes ideal for the adsorption of gas and their application in producing fuel cells. Nanotubes also react to the presence of electrical fields by bending and thus modifying their resonance frequency. This characteristic could lead to applications in the field of nanotechnologies, with the manufacturing of nanoscales and nanometric electromechanical switches. Since nanotubes have all these properties and more, it is not surprising that many commercial products already contain them, and many more are currently being studied, designed, and marketed.

Nowadays, the unzipping of single and multiwalled nanotubes offers a viable route for large-scale synthesis of graphene flakes [19]. Recently, carbon structures with $\mathrm{sp}^{2}$ hybridization, such as bilayer, few-layer graphene, and graphene nanoribbons, have entered the flat nanocarbon world [20]. Each of these systems exhibits peculiar properties which are different from both graphene and graphite [21-23]. However, most properties of graphene films and graphene nanoribbons remain unexplained and, therefore, cannot be controlled without a knowledge of the structural defects such as spontaneous warping of the sheets [24-26].

The mechanical, electrical, optical, chemical, and thermoelectric properties of carbon materials are strongly influenced by the proportion of $\mathrm{sp}^{3}$ carbon atoms in the material. Due to their chemical inertness, high hardness, and low friction, amorphous carbon finds applications in coating for magnetic storage devices, razor blades, optical devices, field emitting devices, and biocompatible coating for human implants [27].

In spite of this wide use of nanomaterials, which will likely further increase, their negative effects on health are not well known. The nanometric size enables these materials to go through any biological barrier. While on the one hand this characteristic can be exploited in biomedical applications, such as drug delivery to target organs $[28,29]$, on the other hand there are considerable concerns that there is a risk that these potentially harmful agents are highly pervasive. In vitro and in vivo studies on cytotoxicity and genotoxicity, together with epidemiological studies on the correlation between concentrations of ultrafine particles and cardiorespiratory diseases, are starting to provide information on the biological effects of nanomaterials [30-38].

The studies on nanomaterials, conducted on cell cultures and laboratory animals, are providing evidence on cytotoxicity and genotoxicity effects, on oxidation stress and on damages of the respiratory, cardiocirculatory, neurological, and immune systems. Sometimes, however, the conclusions drawn by various authors are contrasting. This can partly be attributed to the fact that there are different experimental conditions applied in the tests (e.g., the cell types and animals exposed, the nanomaterials used for exposure, and dosage values). For some of these parameters, control was not easy. An example can be seen in the nanotube samples. If different types are used in the various experiments (single-walled, multiwalled, pristine, purified, functionalized, decorated), there will be different responses from the biological systems. Even if the same type of nanotube is used, there may also be considerable differences between one sample and another. This is all the more so if the nanotubes are bought commercially, in this case one lot might be completely different from another one even if purchased from the same seller. This obviously makes it more difficult to compare the results obtained not only by different laboratories, but also within the same research group, if there has not been highly accurate control of the experimental conditions. The technical characteristics and the biological effects are heavily dependent on the chemical and physical properties of the nanomaterials in general, and therefore, in the specific case, also of nanotubes. It is thus essential, both for their technological application and for evaluating any negative effects on health, to characterize as far as possible the nanostructures from a chemical and physical point of view.

This paper illustrates the characterization by analytical transmission electron microscopy of low-dimensional carbon nanostructures, such as nanotubes for $1 \mathrm{D}$ carbon nanostructures, and amorphous and graphene flakes for $2 \mathrm{D}$ ones.

We applied, in a transmission electron microscope, high resolution imaging, electron diffraction, energy dispersion $\mathrm{X}$-ray spectroscopy (EDXS), and electron energy loss spectroscopy (EELS). The main drawback of these techniques is related to the possible local damage of the sample by the highly focused energetic electron beam (the energy is deposited in a volume of only few $\mathrm{nm}^{3}$ ). Electron induced damages can take a variety of forms, including dissociation, desorption, oxidization, reduction, polymerization, carbonization, and diffusion depending on the specimen material and the gases present in the ultrahigh vacuum system $[39,40]$. Therefore, particular care must be devoted to the investigation of nanostructures, so as to minimize electron beam damaging. This can be obtained using the nanobeam where the electron damage is minimized due to the low beam intensity.

Unconventional nanobeam electron diffraction (NED) and EELS (NEELS) measurements were made using a nanometer-sized coherent parallel beam of electrons [41, 42]. In particular, by reducing the size of the incident electron beam to a few nanometers with the condenser aperture and the spot size condenser lens, EELS spectra and diffraction patterns were acquired on single low-dimensional nanostructures. Unlike conventional selected area electron diffraction (SAED), the diffraction volume is defined directly by the electron probe [43]. Finally, TEM images of the nanostructure under investigation should be taken before and after acquisition to ensure the quality of the measurements.

The purpose was to measure the main size parameters of the nanostructures and to identify the presence of structural defects and of contaminants like the catalyst used to synthesize the nanostructures. 


\section{Materials and Methods}

2.1. Sample Preparation. For observation with TEM microscopy, the nanotubes, the graphene, and the highly oriented pyrolytic graphite (HOPG) flakes were dispersed in isopropyl alcohol. The suspension was placed in an ultrasound bath for about 5 minutes. A few drops of the suspension were deposited on standard size (diameter $3.05 \mathrm{~mm}$ ) 1,000 mesh gold grids. If we had analyzed the length of the nanotubes, we would have placed a few drops of the suspension on a 400 mesh copper grid on which a layer of amorphous carbon has previously been placed. Since the information about the measurement of the length of the nanotubes is of little use, the extremely high ratio between the length and diameter can, for practical purposes, lead to consider the nanotubes as 1 dimensional structures, that is, with an infinite length with respect to the diameter, copper grids with amorphous carbon film were not used. Only the gold grids without any film were used. The nanotubes remain suspended between the bars of the grid, and the absence of a support film enables optimal observation.

Standard $\mathrm{Cu}$ grids covered with amorphous carbon film were used to investigate thin amorphous layers [44].

2.2. Transmission Electron Microscopy. The microscope used (model Tecnai 12 G2 Twin, FEI Company, Hillsboro, OR, USA) was equipped with a thermionic gun (single-crystal lanthanum hexaboride), with an energy dispersive X-ray spectrometer (model Genesis 4000, EDAX Inc., Mahwah, NY, USA) and a "post-column" electron energy filter (Bio filter, GATAN Inc., Pleasanton, CA, USA). The energyfiltered images and EELS spectra were acquired by the use of a slow-scan CCD camera (model 794 IF, GATAN Inc., Pleasanton, CA, USA).

Conventional imaging was performed in energy filtered image mode configuration, at electron energy of $120 \mathrm{keV}$, with a collection angle of about $20 \mathrm{mrad}$. To enhance image contrast and resolution, chromatic aberrations were reduced by collecting only elastic electrons $(\Delta E=0)$. All the high resolution images were collected at the "Scherzer defocus", in order to optimize the transfer function of the optical system balancing the effect of spherical aberration (Cs $=2.2 \mathrm{~mm}$ ) against a particular negative value of $\Delta f$ (about $103 \mathrm{~nm})$. In this case the image contrast in the Weak Phase Object (WPO) approximation represents the two dimensional projection of the crystal potential [45].

All the NEELS and NED experiments were performed on individual nanostructures by using a nanometer-sized (up to about $20 \mathrm{~nm}$ ) coherent parallel electron beam [43]. Actually, these joint measurements allowed us to directly visualize the area of the specimen (about $50 \mathrm{~nm}$ in diameter) from which spectra and diffractions were acquired.

Qualitative elemental analysis was performed by means of EDXS [45].

2.3. Statistical Analysis. To measure dimensional carbon nanotube (CNT) characteristics, conventional TEM images must contain a sufficient number of nanotubes to produce significant statistics, but also sufficiently dispersed and untangled in order to easily obtain the measurement of the diameters, and follow the path of the nanotube so as not to use it again in subsequent measurements. From 4 to 10 TEM images were used to analyse the dimensions, by which it was possible to acquire the values of the diameters of between 60 and 80 nanotubes.

2.4. Electron Energy Loss Spectroscopy. Spectra used to perform EELS microanalysis were acquired in image mode (convergence and collection angle of 0.1 and $20 \mathrm{mrad}$, resp.) and the obtained information was relative to the zone defined by the superposition of the circular spectrometer aperture ( $2 \mathrm{~mm}$ of diameter) and the image. Using this configuration, the size of the analyzed zone depends on the magnification and can be easily varied.

The sample thickness $t_{r}$ can be determined by EELS measurements (low loss region of Figure 11) by calculating the integrated intensity of the elastic peak, $I_{0}$, and that of the first order plasmon features, $I_{p}$, through the following relation:

$$
t_{r}=\lambda \ln \left(\frac{I_{p}}{I_{0}}\right),
$$

where $\lambda$ is the electron mean free path (about $174 \mathrm{~nm}$ in our case) [46].

For EELS microanalysis, ionization edges of the investigated species were analysed. The edge detection was defined by the minimum permissible signal/noise $(S / N)$ ratio as 3 , corresponding to $98 \%$ certainty for detection of the element [47].

Spectral processing for the estimation of the atomic ratios was performed according to the Egerton procedure [48]. In order to calculate the atomic ratio, the following relation was used:

$$
\frac{N_{x}}{N_{y}}=\frac{S_{x}(\alpha, \Delta) \sigma_{y}(\alpha, \Delta)}{S_{y}(\alpha, \Delta) \sigma_{x}(\alpha, \Delta)},
$$

where $S_{x}$ is the integrated core edge intensity for element $x$ in an energy window $\Delta$, with a collecting angle $\alpha$ and $\sigma_{x}, \sigma_{y}$ are the ionization cross-sections calculated from the hydrogenic model $[49,50]$ using the same energy and angle values. In general, a power law $[47,51]$ describes the background under the edge:

$$
B(E)=A E^{-r},
$$

where the two parameters $A$ and $r$ are obtained by the leastsquare minimization in a limited energy region $\delta$ before the edge and extrapolated above the edge region to be integrated. The correlation coefficient between the experimental points in the energy window $\delta$ and the points relative to the chosen background was used as test of fit. For both the C-K edge and the $\mathrm{O}-\mathrm{K}$ edge the widths of the fitting ranges $(\delta)$ were $10 \mathrm{eV}$ and $190 \mathrm{eV}$, respectively, and the width of energy window used to calculate the element relative quantification $(\Delta)$ was $25 \mathrm{eV}$ for both elements.

Error sources in EELS microanalysis have been extensively discussed $[47,52]$. Statistical limitation, approximate 
calculation of the appropriate cross-sections, and, mainly, uncertainty in the background subtraction are the principal sources. The error bar relative to EELS measurements was estimated to be about $20 \%$ when particular problems in the background interpolation are absent, that is; when correlation coefficients between experimental points and background are higher than 0.9 (corresponding to a probability that the two curves are not correlated less than 0.001).

Elemental composition was analyzed by means of Digital Micrograph software (version 3.9.5, Gatan Inc., Pleasanton, CA, USA).

\section{Results and Discussion}

3.1. One-Dimensional Nanomaterials. We intend CNTs as 1-dimensional carbon nanomaterials and several kinds of CNTs were analyzed. Conventional TEM images and high resolution images were acquired at different magnifications values. The quality of the medium-sized magnifications of the conventional images already makes it possible to measure the external and inner diameters of nanotubes and thus to calculate the number of walls of the nanotubes. The highresolution images make it possible to directly measure the number of and the distance between the walls, to evaluate the presence of structural defects and the presence of amorphous carbon on the external walls of nanotubes.

The chemical composition of the nanotubes, of the catalyst nanoparticles, and of those used for decoration was analyzed by EDXS and EELS. Analyzing the fine structure of the EELS spectrum near the carbon $\mathrm{K}$ edge (energyloss near-edge-structure, ELNES), information on the nature of the chemical bonds of the carbon in the nanotubes can be obtained, whereas it is possible to obtain structural information on the nanomaterial by studying the oscillations in the carbon spectrum following the carbon peak (extended energy-loss fine structure, EXELFS) $[53,54]$.

Conventional TEM images with sufficiently high magnifications were acquired to facilitate the measurement of the nanotubes' outer diameters and inner channels. Figure 1 shows TEM images acquired to measure the nanotube diameters: (a) for a sample of undecorated nanotubes; (b) for nanotubes decorated with copper nanoparticles, clearly observed as being attached to the external surfaces. The images clearly show the essential geometric characteristics of the tubes, with darker external walls and the lighter internal channels. All this information cannot be obtained with scanning electron microscopy, which only provides images of the tubular surfaces of the samples, without details on their internal structure (in practice, it is not possible to establish whether the tubes are full or hollow). We remind that decoration with copper nanoparticles enhances the photocurrent response of CNT [55-57].

In Figure 1 a sample of "bamboo-like" nanotubes is also shown. In (c) how it is not correct to talk of 1-dimensional structure because the length of these type of nanotubes can be very short is clearly shown: the nanotube can easily break in a node point. In (d) a detail of a nanotube is shown, with the absence of the inner channel and the axial symmetry.
TABLE 1: Statistics regarding the measurement of external diameters, the inner channel, and the number of walls for undecorated and decorated nanotube samples.

\begin{tabular}{lccc}
\hline Sample & $\begin{array}{c}\text { Outer diameter } \\
(\mathrm{nm})\end{array}$ & $\begin{array}{c}\text { Inner channel } \\
(\mathrm{nm})\end{array}$ & Wall number \\
\hline Undecorated & $7.6 \pm 1.7(50 \%)$ & $4.0 \pm 1.0(37 \%)$ & $5 \pm 1(23 \%)$ \\
nanotubes & $10.8 \pm 3.0(44 \%)$ & $4.9 \pm 3.3(63 \%)$ & $9 \pm 1(35 \%)$ \\
& $15.4 \pm 1.5(6 \%)$ & & $5 \pm 1(42 \%)$ \\
\hline & $7.9 \pm 2.2(33 \%)$ & & $8 \pm 1(29 \%)$ \\
Decorated & $11.5 \pm 2.2(37 \%)$ & $4.0 \pm 2.3(74 \%)$ & $9 \pm 2(41 \%)$ \\
nanotubes & $14.5 \pm 1.7(23 \%)$ & $7.1 \pm 2.4(26 \%)$ & $13 \pm 2(21 \%)$ \\
& $17.2 \pm 4.1(7 \%)$ & & $13 \pm 0$. \\
\hline
\end{tabular}

The errors associated with the value of the diameters and walls are equal to a standard deviation.

Unlike the others, the micrographs shown in (c) and (d) were acquired using a 400 mesh copper grid coated with a carbon film, due to their limited length.

Table 1 shows statistical data on the measurement of the outer diameters, inner channels, and the number of walls for both samples, undecorated and decorated. The data were obtained by showing a histogram of the number of times the value of the outer diameter of the nanotubes has been counted, for example, as shown in Figure 2; this number must be in $1 \mathrm{~nm}$ intervals and fit with a suitable Gaussian number. The values shown in Table 1 are equal to the maximum of the Gaussian curve, with the associate errors equal to one sigma and the percentages in parentheses being obtained in such a way that the sum of the areas included in the Gaussian curves is equal to 100 .

Using high resolution images, we can directly count the number of nanotube walls, measure the distance between the walls, and verify the presence of structural defects in the nanotube, such as the closing of the inner channel, the breakage of one or more walls, the presence of amorphous or crystalline carbon on the external walls, and the presence of nanoparticles of the catalyst and of materials used for internal and external decoration of the nanotubes. To perform both high resolution TEM (HRTEM) images and spectroscopic analysis we chose to self-support CNTs on mesh grids, without any additional support films. In this way we avoid the interferences due to any substrate on images and spectra. The presence of a carbon film, however, thin it may be, could worsen the contrast obtainable for the photographs and interfere with the nanotubes' EELS analysis.

Figure 3 shows some examples of HRTEM images of nanotubes with different numbers of carbon walls. In (a) a bundle of single-wall nanotubes, in (b) a three-wall nanotube (as an example of few-wall nanotube), and in (c) a nanotube with more than ten walls (as an example of many-wall nanotube) are shown. All the nanotubes have very few defects. In (b) and (c) it is possible to directly observe pieces of single graphene sheets onto the external surface of the nanotubes [24].

By HRTEM it is possible to accurately study CNTs defects at the atomic scale. Some very common nanotube defects can be observed in Figure 4(a). The inner channel is not well defined and is characterized by variable diameter. In 


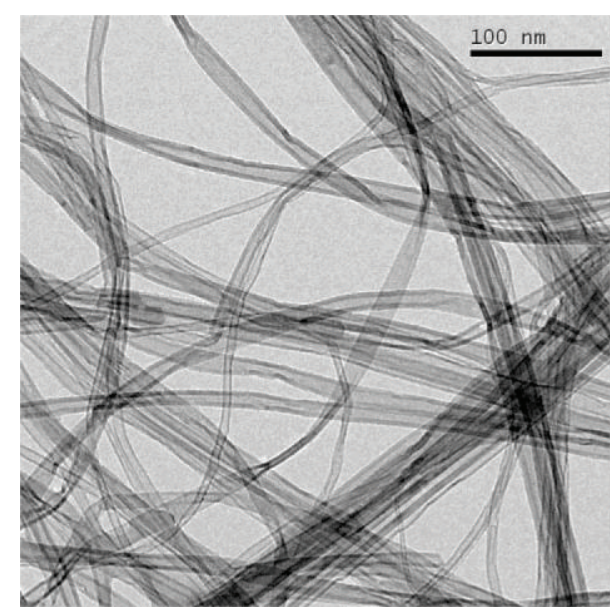

(a)

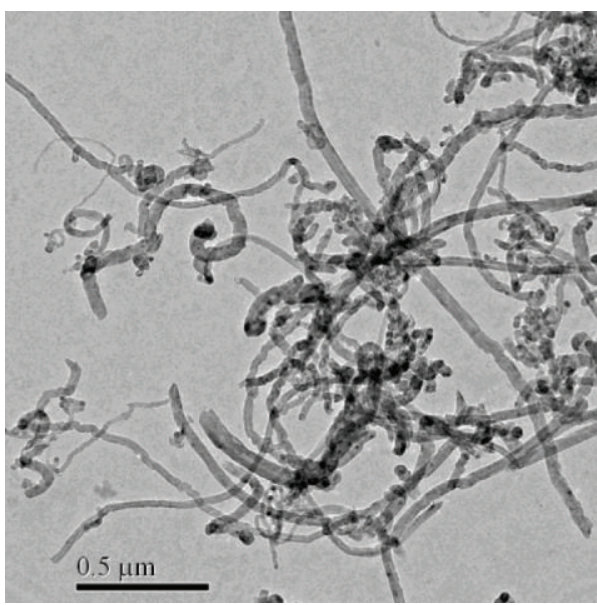

(c)

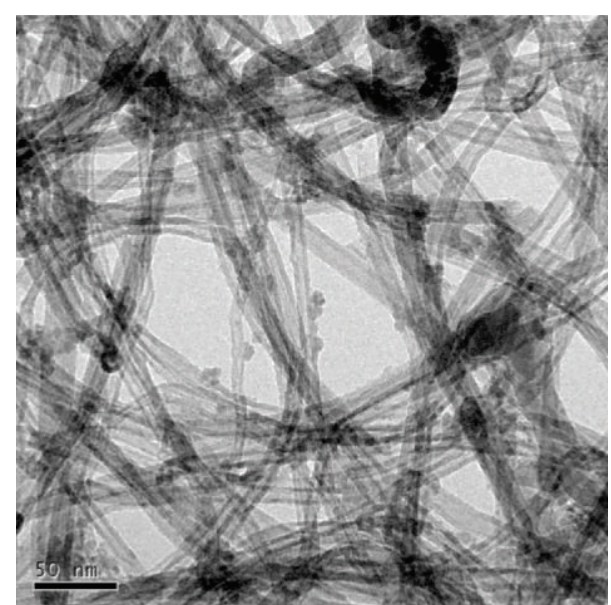

(b)

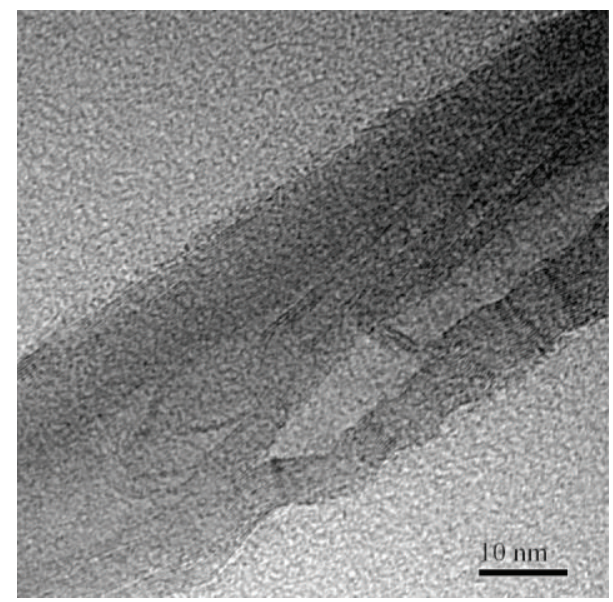

(d)

FIGURE 1: TEM images of (a) nondecorated nanotubes; (b) decorated nanotubes; (c) "bamboo-like" commercial nanotubes; (d) high resolution image of a bamboo-like nanotube.

many points the channel is broken by the carbon walls and onto the external nanotube surface numerous pieces of single graphene sheets crumpled up can be directly visualized. By HRTEM less common defects can also be visualized. Figure 4(b) shows a rupture of the carbon sheets is normal to the nanotube axis. Figure 4(c) shows a particular nanostructure where the internal channel was not created at all and the nanomaterial resembles a stack of "nanocups."

Finally, a special mention for Figure 4(d) showing a very particular form of a spiral structure, in a way that we could speak of a carbon "nano-spring." the number of carbon atoms linked in hexagonal rings with respect to that linked in pentagonal rings is such that the nanotube assumes the spiral configuration.

The composition of the elements of the nanotubes and nanoparticles can be analysed by EDX spectroscopy in a wide sample zone (of about $500 \mathrm{~nm}$ ) including several CNTs and nanoparticles. Figure 5(a) shows an EDXS spectrum of carbon nanotubes while Figure 5(b) shows that of catalyst nanoparticles $(\mathrm{Fe})$ and decoration material $(\mathrm{Cu})$. The Au peak is due to the grid and though it is very intense, it does not interfere with the sample peaks.

EELS spectroscopy allowed us to select single nanostructures and acquire C-K edge spectra as reported in Figure 6(a). One of the greatest potentials of EELS spectroscopy is the exact spatial localization of the acquired data. Indeed, with special apertures, it is possible to acquire the spectra coming only from a selected portion of the sample, around $50 \mathrm{~nm}$. As an example, in Figure 6(a) (inset) the portion of the nanotube, from which the spectra were acquired, is shown.

Figure 6(a) shows the $\mathrm{C}-\mathrm{K}$ edge, where the near edge fine peaks are clearly visible located at $284 \mathrm{eV}$ and $290 \mathrm{eV}$ that can be ascribed to the carbon molecular orbitals $\pi^{*}$ and $\sigma^{*}$, respectively. This $\mathrm{C}-\mathrm{K}$ edge resulted to be well structured, indicating the highly ordered carbon atomic arrangement of the CNT.

Figure 6(b) shows an EELS spectrum with the C-K, O-K, and $\mathrm{Fe}-\mathrm{L}_{2,3}$ edges. It is important to detect the presence of $\mathrm{O}$ to assess whether the sample has undergone an oxidation process, and if so, how much. Even the occurrence of 


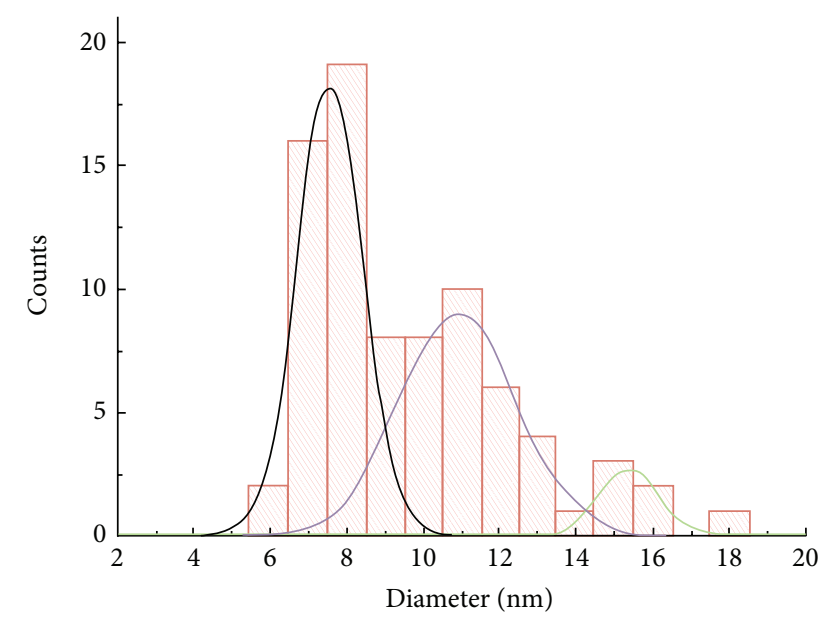

FIGURE 2: Histogram of the measurements of the outer diameter of the undecorated nanotube sample.

nanoparticles on the CNTs surface can be monitored by EELS spectroscopy that can furnish the nanoparticle chemical composition (Figure 6(b)). In this case, the percentage of $\mathrm{C}$, $\mathrm{O}$, and $\mathrm{Fe}$ atoms, obtained by the analysis of the $\mathrm{C}-\mathrm{K}, \mathrm{O}-\mathrm{K}$, and $\mathrm{Fe}-\mathrm{L}_{2,3}$ edge electron energy loss spectrum, are $\mathrm{N}_{\mathrm{C}}$ (at\%) $=94 \pm 13, \mathrm{~N}_{\mathrm{O}}($ at $\%)=5 \pm 1$, and $\mathrm{N}_{\mathrm{Fe}}($ at $\%)=1.0 \pm 0.5$.

EELS spectra contain not only compositional information but even important structural information. In particular, EXELFS spectroscopy is a very powerful technique because with suitable Fourier analysis it is possible to obtain information on the spatial distribution of the first-neighbour atoms with respect to the excited atom, just like the data obtained by spectroscopy using X-rays (extended X-ray absorption fine structure, EXAFS); however, in order to use the last one, a synchrotron is required, and in any case it provides information mediated over the entire sample [58, 59]. Moreover, EXELFS spectroscopy gives structural information along the direction defined by the transferred momentum vector $\mathbf{q}$ which is defined by the acquisition geometry. This is important in the case of anisotropic materials such as graphite or low-dimensional carbon materials. In our experiments we acquired spectra with a wide collection angle $(20 \mathrm{mrad})$, giving rise to a $\mathbf{q}$ vector mainly oriented normal to the electron beam [60].

Figure 7(a) shows the EXELFS and EXAFS oscillations obtained from C-K spectra of the type shown in Figure 6, but for a sample of HOPG. These spectra were compared with the oscillations obtained from a theoretical simulation of the structure of the graphite, using a FEFF v. 8.2 software program [61]. The HOPG is used because it is a material especially suited for comparison with multiwalled carbon nanotubes (MWCNT). In Figure 7(a), it can be clearly observed that the two different techniques-the other using electrons (EXELFS) and the one using X-rays (EXAFS) for exciting the carbon atom in the graphite-reproduce in the same way the energy position of the spectral structures, although the intensities are slightly different. These spectral structures, regardless of the way they were generated, are closely correlated to the structural parameters of the graphite. It can also be noted that the experimental data, which are very similar to each other, are in agreement with the theoretical simulation calculated with FEFF, considering a sphere of graphite with a radius of about $8 \AA$. The corresponding Fourier transforms, Figure 7(b), allow tracing the graphite in terms of distances between atoms. Indeed, if considering that the excited atom corresponds to zero $(R=0)$, the positions of the neighbouring atoms correspond to peaks in the radial distribution function $F(R)$ in the direction defined by $\mathbf{q}$.

The radial distribution of the graphite plane, both for EXAFS and EXELFS, is very well reproduced. Except for any phase factors, it can be concluded that

(i) the first peak represents the first neighbours at $1.42 \AA$,

(ii) the second peak represents the ones at $2.46 \AA$ and $2.83 \AA$,

(iii) the third one includes all the atoms at a distance from the excited atom between $3.35 \AA$ and $4 \AA$.

This result is confirmed by the theoretical simulation performed by FEFF (blue curve).

Figure 8 shows our experimental C-K spectrum concerning a MWCNT. The EXELFS extraction can only be performed with the absence of an O peak, since this would interfere with the extended oscillations of the carbon, making it virtually impossible to process the data. It can be observed that it is very difficult to detect the oscillations in the part of the curve with the highest energies by sight inspection only.

In particular, Figure 8(b) shows the comparison between the EXELFS oscillations of the HOPG and of the MWCNT. With regard to the latter, it is important to note that EXELFS spectrum of MWCNT shows no traces of oxygen $(530 \mathrm{eV})$ and nitrogen $(410 \mathrm{eV})$; this characteristic leads to believe that they are well-graphitized tubes, since nitrogen and oxygen can create structural defects in the walls of the tube. It can also be deduced that in this sample of multiwalled nanotubes there are no metallic precursors such as iron $(720 \mathrm{eV})$, utilized in the synthesis process. The Fourier transforms in Figure 8(c), calculated on the basis of the spectrum in Figure 8(a), highlight that the two structures of the graphite and multiwalled nanotubes are quite similar but not identical $[53,62]$. The results reported here are relative to the atomic structure along the CNT axis, due to the $\mathbf{q}$ orientation in our experimental conditions. What has been described up to now shows in particular the enormous potentials of this technique for the study of the structure in terms of inter-atomic distances around an excited atom.

Figure 9 shows the results concerning the NED experiment on two MWCNTs: a 7-wall versus a 14-wall nanotube. Insets report the relative diffractograms, obtained by the same technique and published by Zuo et al. for a 2 walls nanotube [63]. As it can be observed in the insets, when the number of walls decreases the diffraction spots that lose their circular symmetry and become undefined smears. This feature is in good agreement with the case of 2 walls where Zuo et al. observed wide lines organized in hexagonal symmetry [63]. In our case, we observe the same behaviour, coming from 


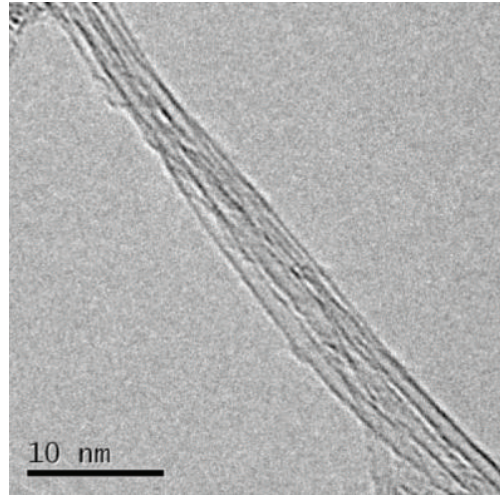

(a)

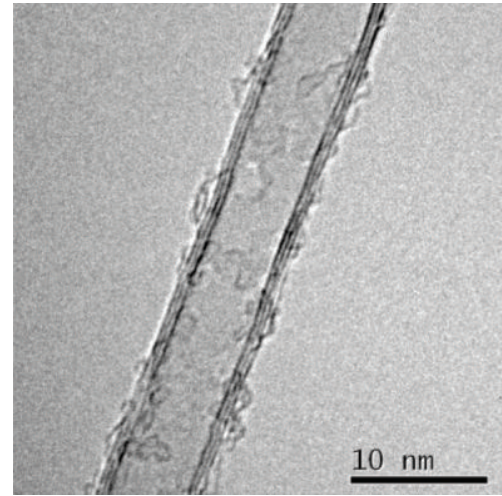

(b)

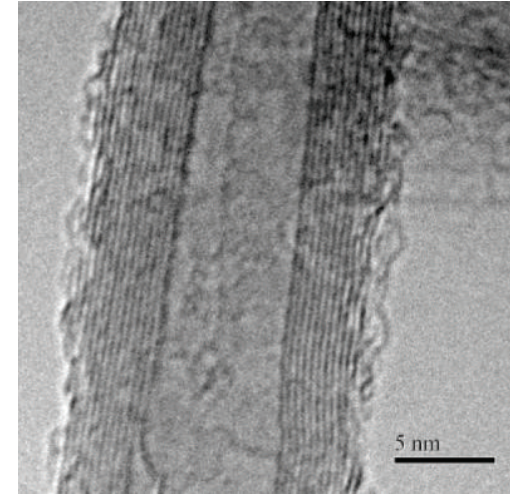

(c)

FIGURE 3: Examples of high resolution TEM images of carbon nanotubes with different numbers of walls: (a) bundle of single-wall nanotubes; (b) three-wall nanotube; (c) multiwall nanotube.

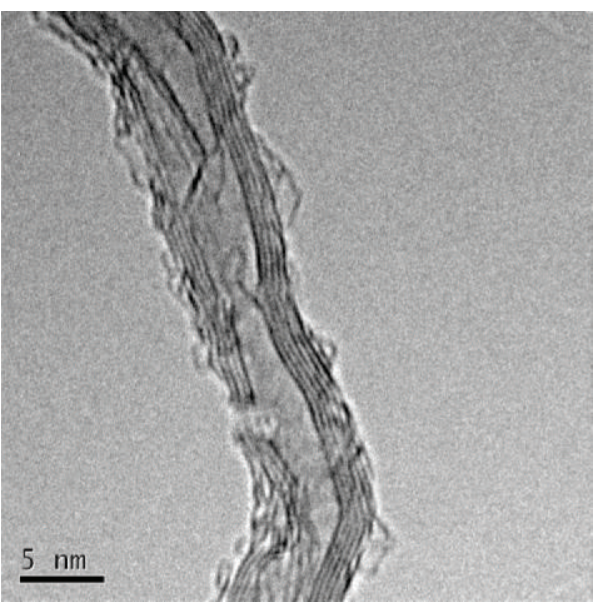

(a)

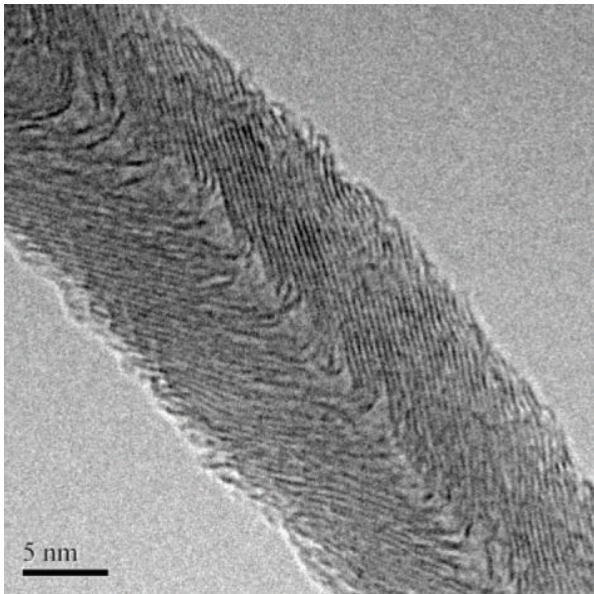

(c)

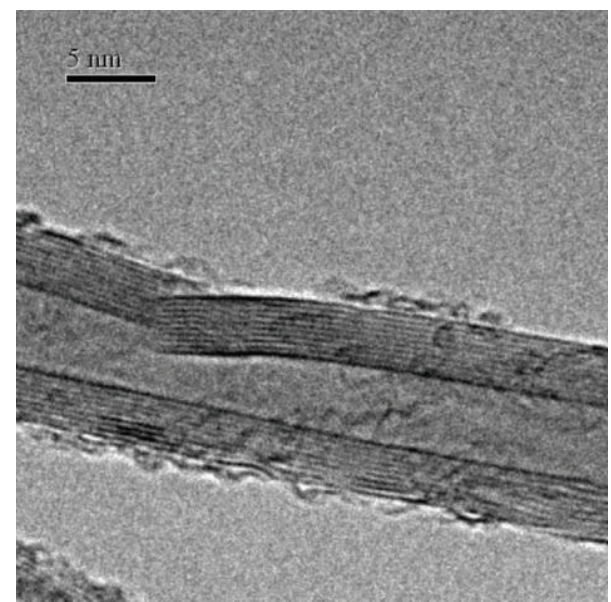

(b)

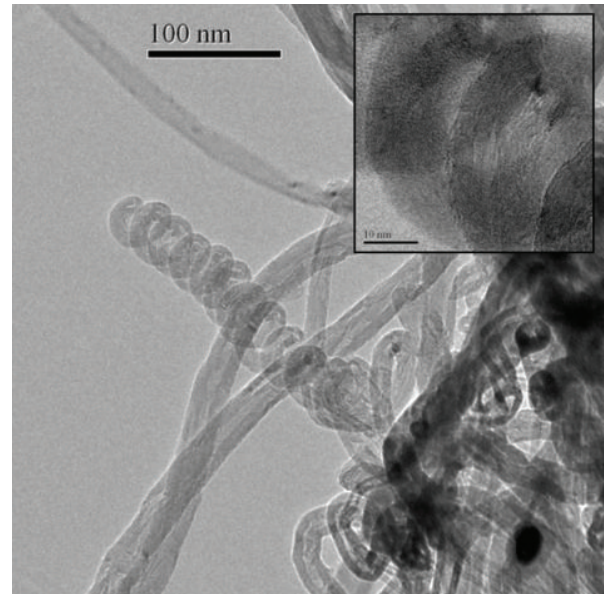

(d)

FIGURE 4: Examples of carbon nanotubes defects: ((a) and (b)) nanotube wall breaks and presence of amorphous carbon and graphene pieces onto the nanotube external surface; (c) a "nano-cup"; (d) a "nano-spring" (the inset shows the high resolution spring coils). 


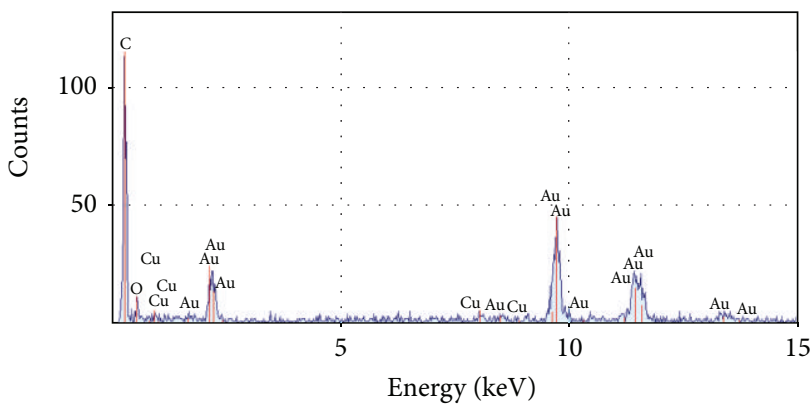

(a)

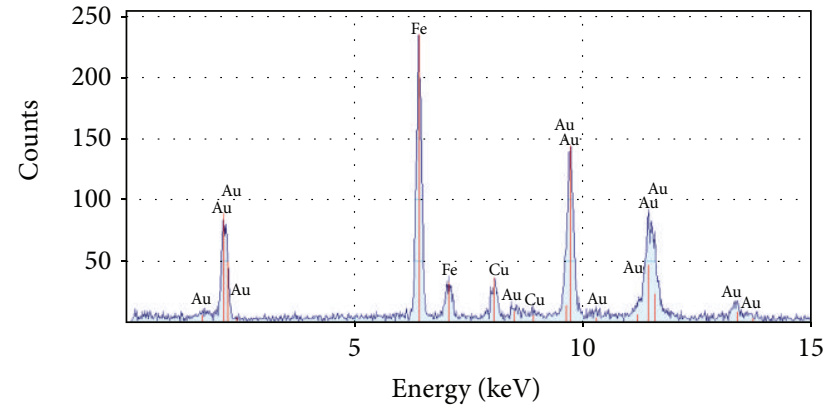

(b)

Figure 5: EDX spectra of: (a) carbon nanotubes; (b) nanoparticles of catalyst (Fe) and decoration material (Cu).

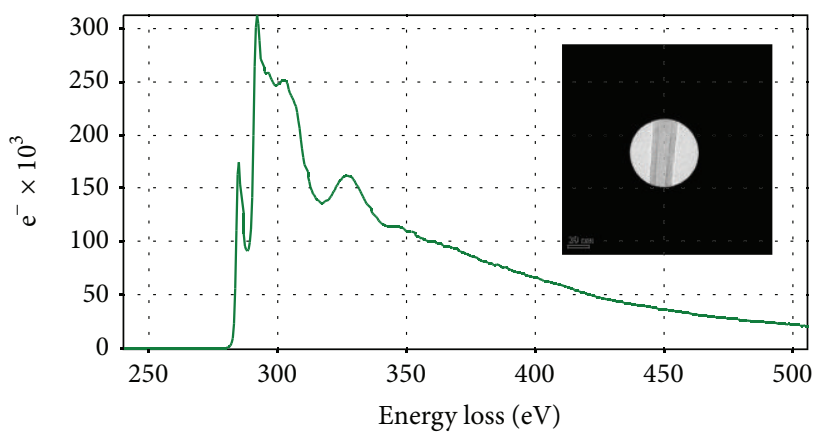

(a)

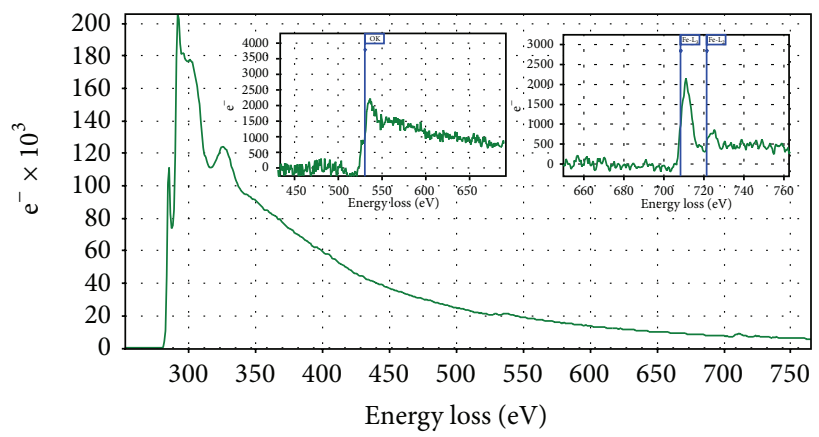

(b)

FiguRE 6: ELNES (a) and EXELFS (b) spectra for the carbon K edge of the undecorated sample. In the inset the portion of nanotube from which ELNES spectrum was acquired is shown. Spectra evidence of the carbon $\mathrm{K}$ edge $(284 \mathrm{eV})$, the oxygen $\mathrm{K}$ edge $(532 \mathrm{eV})$, and the iron $\mathrm{L}_{2-3}$ edge $(708 \mathrm{eV})$.

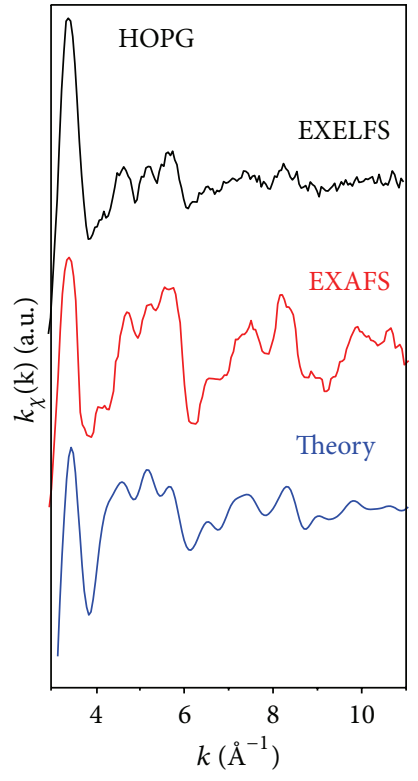

(a)

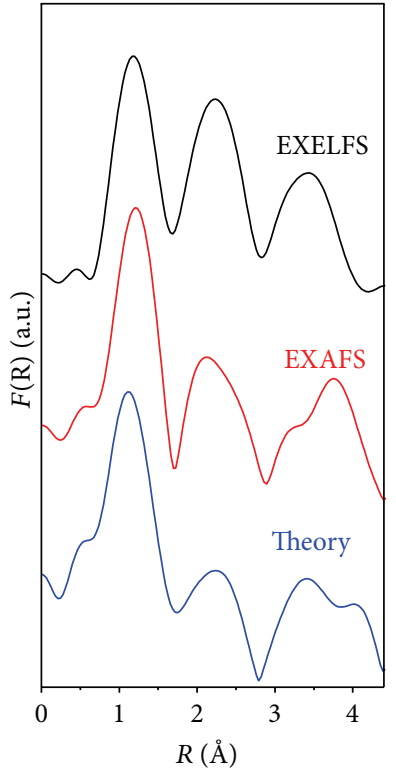

(b)

Figure 7: (a) Comparison of the oscillations of the EXELFS and EXAFS spectra and of the theoretical curve, for HOPG (a) and (b) the corresponding Fourier transforms. 


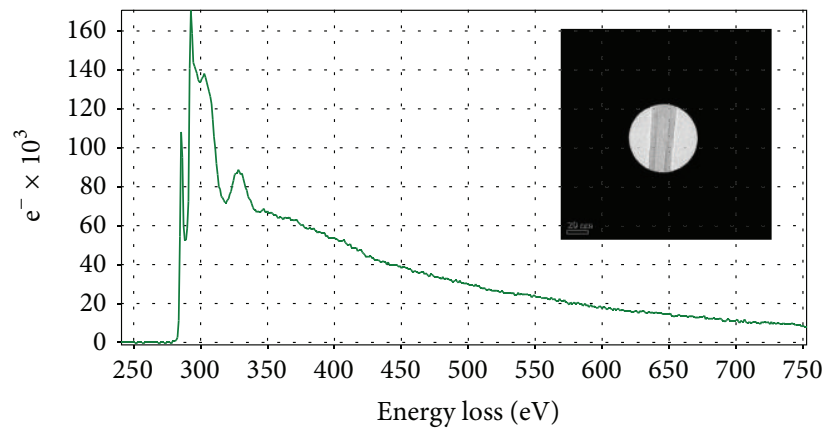

(a)

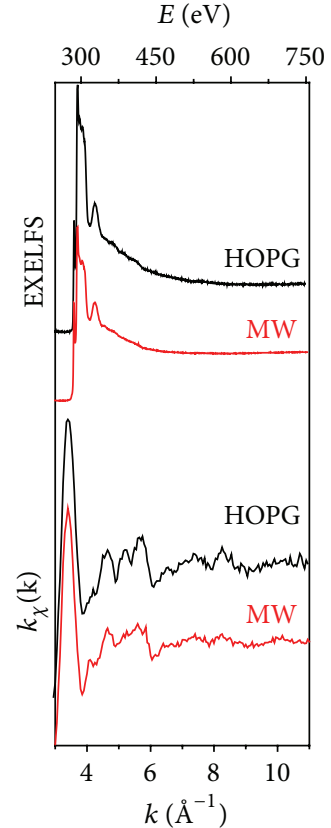

(b)

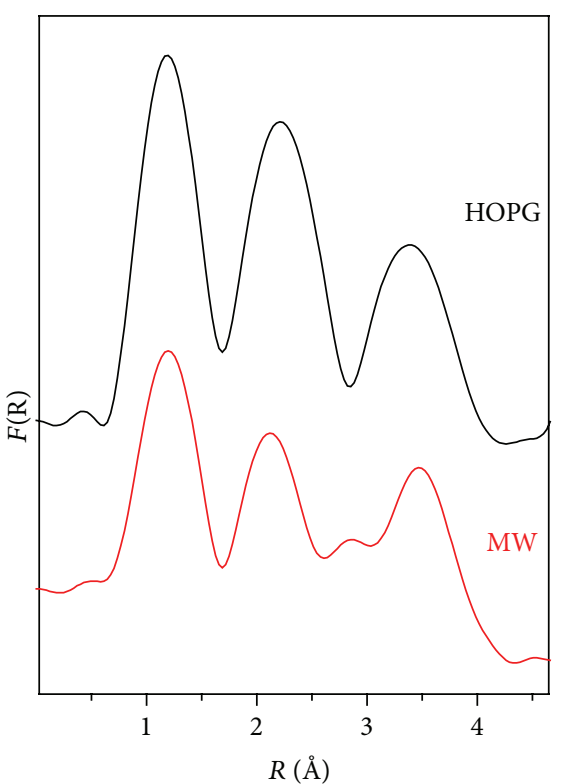

(c)

FIGURE 8: EXELFS of an undecorated MWCNT (a), comparison of HOPG and the MWCNT of the EXELFS spectra and their processing to highlight the oscillations (b), and of the corresponding Fourier transforms (c).

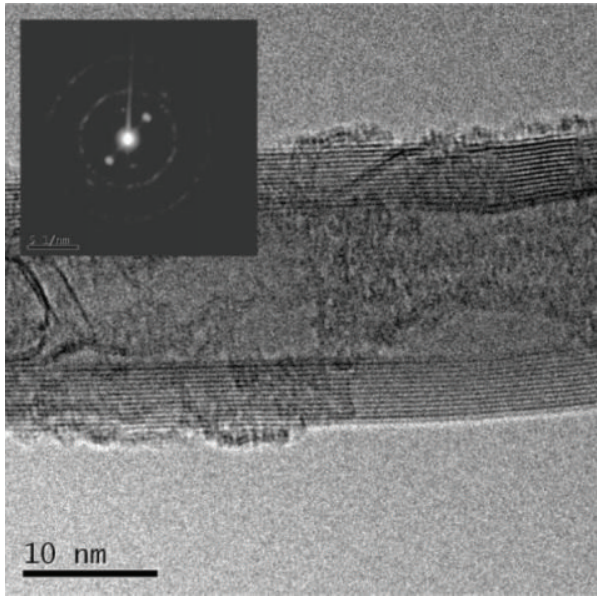

(a)

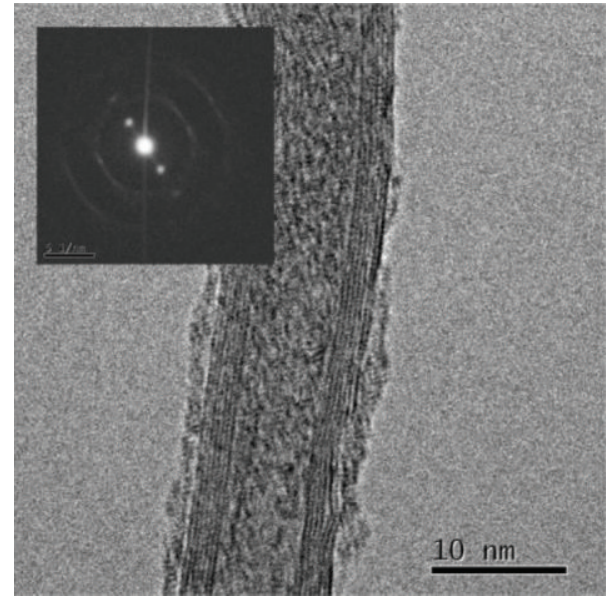

(b)

FIGURE 9: HRTEM images of a 14-wall CNT (a) and a 7-wall CNT (b). The insets show the relative NED patterns. 


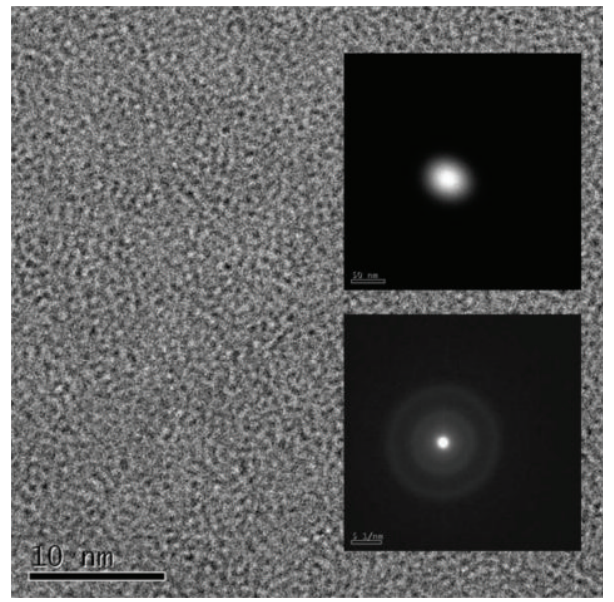

Figure 10: TEM image of a thin amorphous carbon film. The upper inset shows the electron beam spot size employed to obtain the nanodiffraction shown in the lower inset.

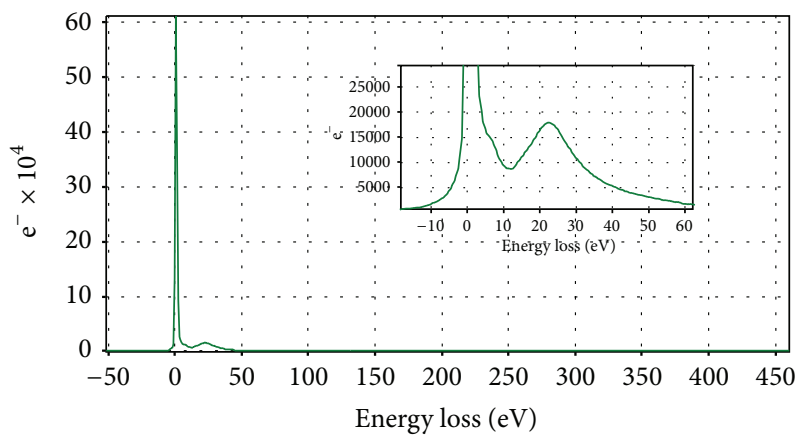

(a)

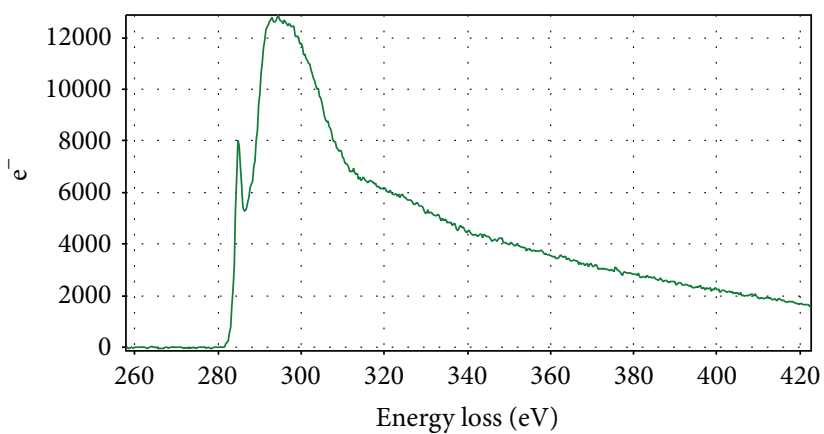

(b)

FIGURE 11: EELS spectra of the amorphous carbon film: (a) zero-loss and low-energy peaks employed to calculate the film thickness; ELNES carbon $\mathrm{K}$ edge obtained in imaging mode (b).

a circular diffraction pattern with spots for 14 walls CNT to an hexagonal pattern with undefined lines for 7 walls CNT.

We stress that only the combined application of EXELFS and NED techniques can give unique structural information about nanomaterials made of few carbon atoms not disposed in ordered symmetries as in a perfect crystal.

3.2. Two-Dimensional Nanomaterials. Self-sustaining, very thin flakes of amorphous carbon and graphene were investigated up to the formation of the graphite structure. With the aim to gain information about their thickness, morphology, defects, elemental composition, and atomic structure we performed both conventional and HRTEM imaging, X-ray and EELS microanalysis, and NED and NEELS experiments.

Figure 10 shows the flat morphology of a thin amorphous film. HRTEM was unable to visualize any structure and only a fine grain can be observed. This is the reason why this kind of carbon material is generally used as substrate in conventional transmission electron microscopy. The fine grain is due to the local order existing in the $\mathrm{C}$ atomic distribution that renders the sample not ideally amorphous. This fact is also observed in the NED pattern reported in the inset, where two very wide and fair Debye rings are visible. Microanalytical results obtained by both EDXS and EELS did not show any contamination or oxidation (data not shown). In the case of Figure 11, we obtained a thickness value of $T=15 \mathrm{~nm}$. Figure 11(b) shows the C-K edge from this amorphous carbon flake where, contrary to the case of CNT of Figure 6, only the peak located at $284 \mathrm{eV}$, ascribed to the $\mathrm{C}$ molecular orbital $\pi^{*}$, is clearly visible. Also the NES resulted to be less structured, in agreement with the quasiamorphous atomic arrangement.

In Figure 12 crumpled graphene flakes, obtained by open single wall nanotubes and imaged at low magnification, are reported.

In Figure 13(a), we report a HRTEM image of a graphene flake, compared to the area illuminated by the electron nanobeam (the bright area in the dark background shown in panel (b)). The diffraction pattern was recorded from exactly the same area as the HRTEM image in panel (b) and under identical illumination conditions. The small beam size makes it possible to probe small volumes of the specimen and, hence, to observe features induced by defects with high spatial resolution. For instance, in region 1 (circled) of Figure 13(a), the diffraction pattern shows twin sixfold symmetric spots (Figure 13(c)) corresponding to a couple of flat layers with 


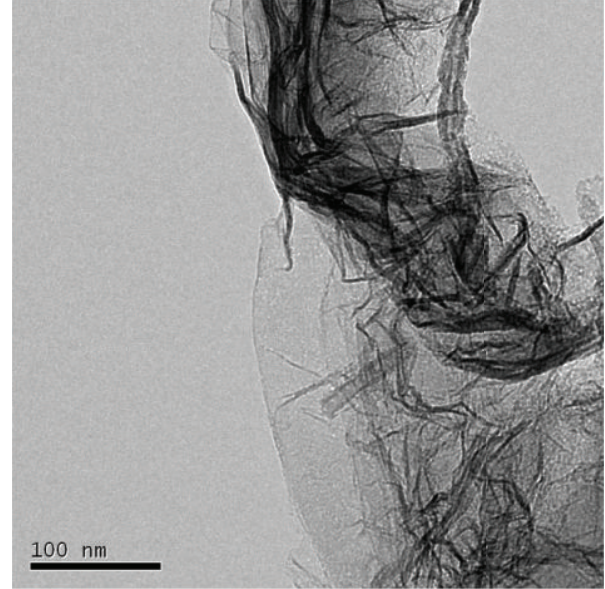

FIGURE 12: TEM image of graphene flakes.

different stacking. The inner spots correspond to (10) lattice planes and the outer spots to (11) planes [24]. The spot separation indicates the good crystalline structure of the layers [64]. In region 2 of Figure 13(a) in which extensive folding of sheets is present, the diffraction pattern is markedly changed. Here, the $\mathrm{c}$-axis is locally tilted from the incident beam direction and the sequence of layers along the c-axis is imaged. The sheet periodicity induces two closely spaced peaks in Figure 13(d) from which an interlayer spacing, $d_{c}$, is estimated [64]. Moreover, Debye rings due to the lack of crystallographic orientation among the sheets are also present.

Depending on the degree of folding, some diffraction patterns have intermediate features between those of Figures 13(c) and 13(d). An example is given in Figure 13(e) in which the sixfold symmetry of (10) and (11) spots indicates a higher degree of ordering than panel (d). We stress that the observed changes are completely averaged out using conventional SAED, due to the lack of spatial resolution. Since the thinnest flakes are just a few tens of nanometers wide, the beam intensity in SAED is too low to record a diffraction pattern from them.

Figure 14 shows low-loss spectra recorded by the nanobeam technique from the same area of the NED reported before. In this region the energy loss is mainly due to the plasmon excitation occurring in the material. Collective excitation of the free electrons strongly depends on the geometry of the samples and, in the case of $2 \mathrm{D}$ nanomaterials, the shape and intensity of the plasmon can give useful information about the sample thickness.

The position of plasmon modes indicates a double layer, as found with diffraction. Moreover, for the two thinnest spectra, the plasmon resonance shows a plateau-like shape (Figure 14(a)), as expected for systems with less than 5 layers [65].

By probing areas of increasing thickness, we observe a continuous shift of the plasmon peaks towards the graphite values and a progressive increase of their intensity. The correlation between the shift and the relative thickness $t_{r}$ is shown in Figure 14(b) in which the position of
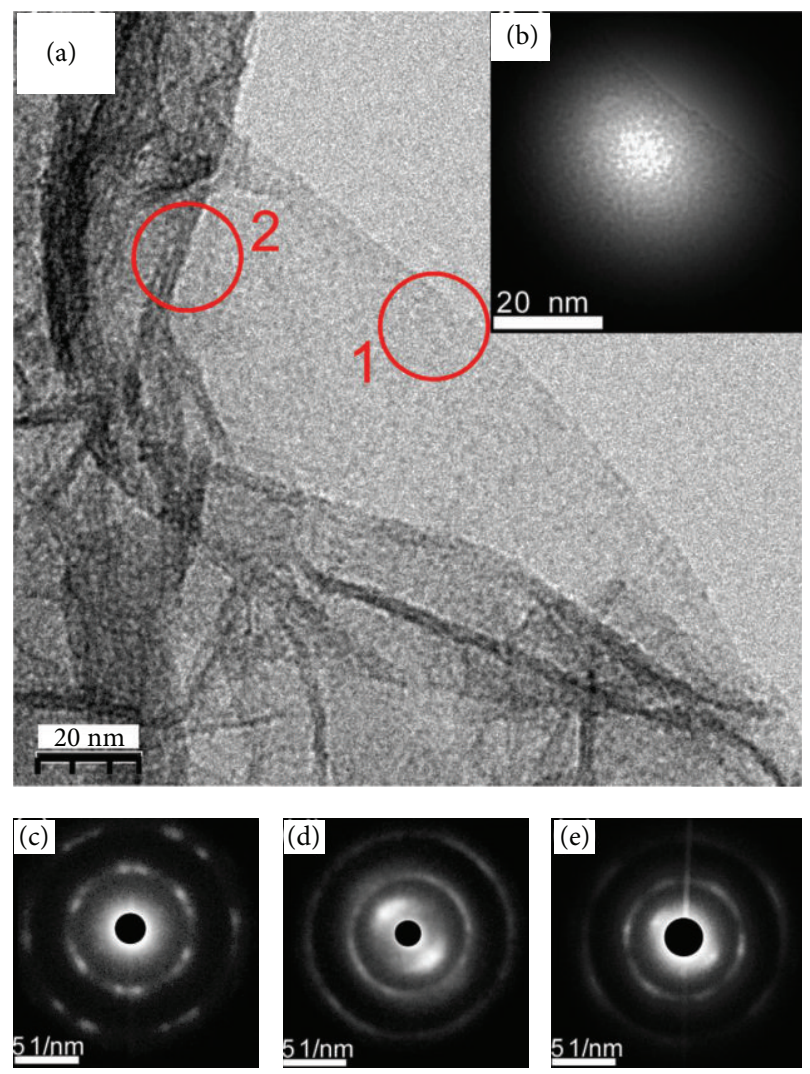

FIGURE 13: (a) High resolution TEM image of a graphene flake compared to the area illuminated by the electron nanobeam shown in panel (b). (c)-(e) NED patterns of different regions of the flakes. Panel (c) is recorded from the area labeled as 1 in panel (a), while panel (d) from the region 2. Panel (e) corresponds to an area of the sample (not shown) having intermediate folding. Reprinted with permission from [64].

the $\pi$-resonance is plotted as a function of $t_{r}$. If we assume, in accordance with electron diffraction data, that the thinnest sample is a double layer of graphene, the absolute thickness can be estimated by the calibration curve of Figure 14(b). It is worth noting that conventional analysis, based on EELS data only, would have overestimated $t_{r}$ in this range of thickness, as it generally occurs for very thin films [47].

Finally, in Figure 15(a) a well-formed HOPG flake is reported, together with the relative nanobeam diffraction pattern and $\mathrm{C}-\mathrm{K}$ edge spectrum. In this case, the thickness is $84 \mathrm{~nm}$. In Figure 15(a), at low magnification, the superposition of multiple layers is indicated by the different gray levels. The inset shows the NED hexagonal pattern, demonstrating that the flake c-axis is parallel to the beam. In Figure 15(b), the C-K edge is reported. At this stage, the material cannot be considered bidimensional, but it is now a perfect tridimensional crystal.

\section{Conclusions}

The data obtained confirm that transmission electron microscopy is a suitable technique for the characterization 


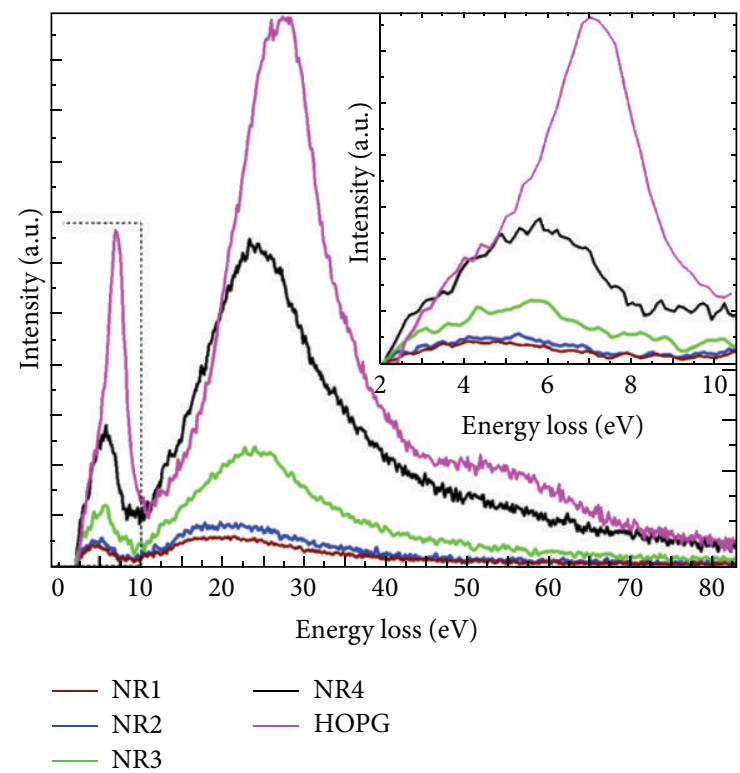

(a)

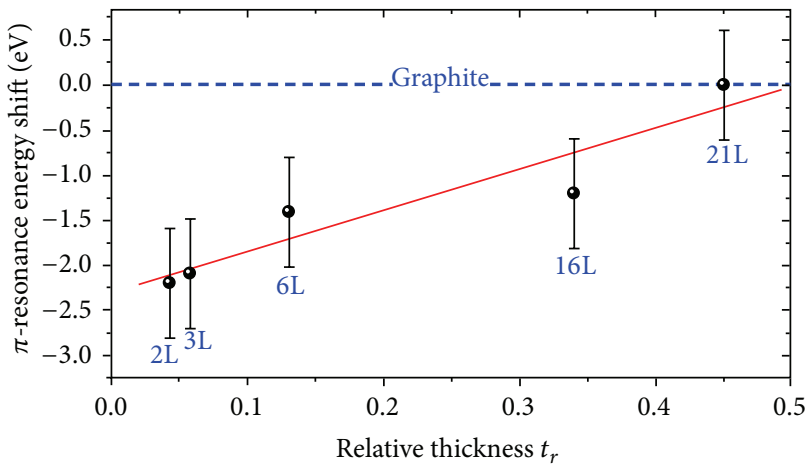

(b)

FIGURE 14: (a) EELS spectra obtained from graphene flakes of different thicknesses and from graphite. In the inset, the $\pi$ resonance peaks are blown up. (b) Energy shift of $\pi$-resonance as a function of the relative thickness $t_{r}$ obtained from the EELS spectra with the log-ratio method. The number of layers $(\mathrm{L})$ is estimated by calibrating the EELS thickness data to the results of electron diffraction, assuming a linear dependence of $t_{r}$ on the number of layers. Reprinted with permission from [64].

of low-dimensional carbon nanostructures, due to the possibility of providing morphological, structural, and elemental composition information. Likewise, with respect to the other techniques for the characterization of nanostructures that are also highly useful, for example, Raman spectroscopy, electron microscopy enables to obtain data with accurate spatial localization on limited zones of nanostructures (few nanometers), and not just on massive samples of micrometric or larger sizes, in which the information inevitably comes from a large number of objects, in which there may be unwanted contaminants that may generate signals that irretrievably alter the data coming from the sample under analysis.

The use of EELS allows to obtain highly localized spectroscopic and structural information, but it is somewhat

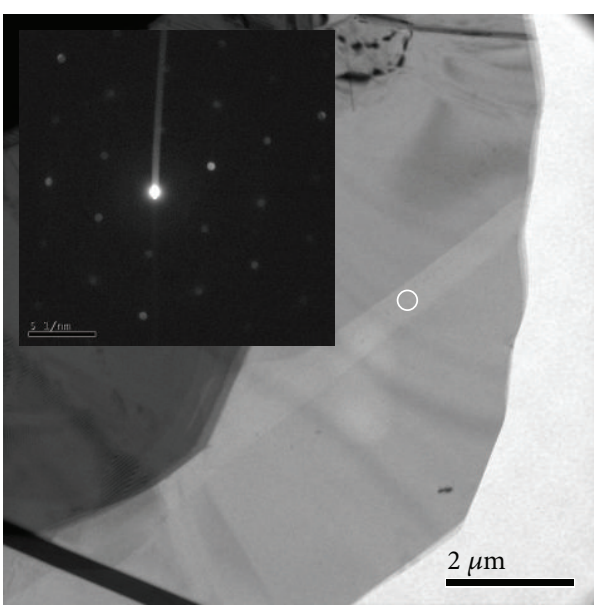

(a)

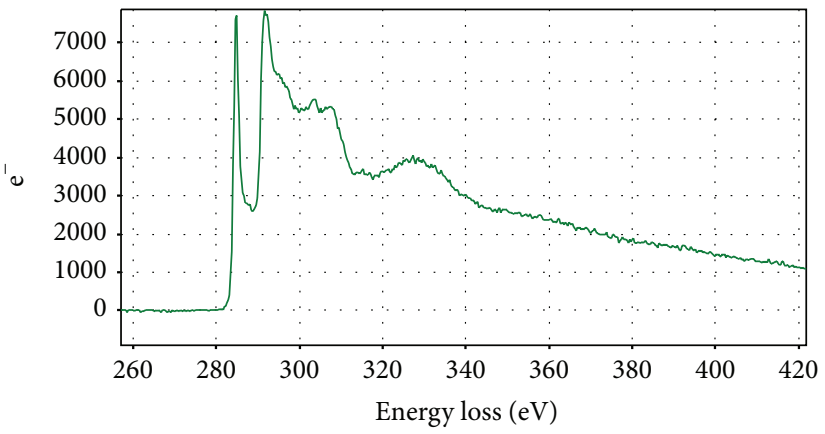

(b)

FIGURE 15: TEM image of a HOPG flake (a). The white circle shows the point from where the NED (inset) and the C-K edge spectrum (b) are taken.

limited by the low detection efficiency for heavy elements, the intrinsic difficulty of the technique in supplying quantitative information on the amount of elements contained in the sample, and by the highly specialized skills required for performing ELNES and EXELFS analysis.

The importance of being able to correctly characterize low-dimensional carbon nanomaterials will undoubtedly increase due to the increasing use and dissemination of these materials. Characterization is a necessary step also for developing synthesis processes. As far as accurate the checking of the parameters may be (e.g., in the case of chemical vapour deposition, the substrate temperature, the vacuum level, the pressure of the gaseous precursor, and the sizes of the catalyst), the nanotubes synthesized in different processes will necessarily have differing characteristics. This is an aspect familiar to those who, for example, purchase commercial nanotubes which, though bought from the same supplier, are completely different from one lot to the next.

Indeed, in various cases, because of the analyses conducted with TEM microscopy, it has been ascertained that the synthesis process of the commercial nanotubes had not been properly performed, with the consequent production of nanotubes full of structural defects, often of the bamboo-like type, with a different configuration with respect to the typical 
ones of a nanotube, that is, without a defined number of walls, a uniform outer diameter, and an inner channel. Characterization is likewise fundamental for correctly assessing the influence that nanomaterials can have on biological systems, since the presence of any negative effects is closely related to the type of nanostructure involved.

Finally, the analysis of the airborne particulate is increasingly affected by the presence of nanostructural materials intentionally produced by man, which are dispersed in working and living environments. Electron microscopy, with its powerful analytical instruments, proves particularly useful in the characterization of ultrafine particles in which there is a wide variety of structures, differing in composition, size, state of aggregation, and origin. These investigations are fundamental both to correlate the presence of biological effects on man with real harmful agent and to identify manmade and other sources most responsible for the production of nanometric airborne particulate.

\section{Conflict of Interests}

The authors report no conflict of interests.

\section{References}

[1] S. Iijima, "Helical microtubules of graphitic carbon," Nature, vol. 354, no. 6348, pp. 56-58, 1991.

[2] A. Oberlin, M. Endo, and T. Koyama, "High resolution electron microscope observations of graphitized carbon fibers," Carbon, vol. 14, no. 2, pp. 133-135, 1976.

[3] A. Oberlin, M. Endo, and T. Koyama, "Filamentous growth of carbon through benzene decomposition," Journal of Crystal Growth, vol. 32, no. 3, pp. 335-349, 1976.

[4] P. Avouris, T. Hertel, R. Martel, T. Schmidt, H. R. Shea, and R. E. Walkup, "Carbon nanotubes: nanomechanics, manipulation, and electronic devices," Applied Surface Science, vol. 141, no. 3-4, pp. 201-209, 1999.

[5] C. Dekker, "Carbon nanotubes as molecular quantum wires," Physics Today, vol. 52, no. 5, pp. 22-28, 1999.

[6] A. D. Maynard, P. A. Baron, M. Foley, A. A. Shvedova, E. R. Kisin, and V. Castranova, "Exposure to carbon nanotube material: aerosol release during the handling of unrefined single-walled carbon nanotube material," Journal of Toxicology and Environmental Health A, vol. 67, no. 1, pp. 87-107, 2004.

[7] O. V. Salata, "Applications of nanoparticles in biology and medicine," Journal of Nanobiotechnology, vol. 2, article 3, 2004.

[8] R. Beckman, E. Johnston-Halperin, Y. Luo, J. E. Green, and J. R. Heath, "Bridging dimensions: demultiplexing ultrahigh-density nanowire circuits," Science, vol. 310, no. 5747, pp. 465-468, 2005.

[9] J. Y. Chen, A. Kutana, C. P. Collier, and K. P. Giapis, "Materials science: electrowetting in carbon nanotubes," Science, vol. 310, no. 5753, pp. 1480-1483, 2005.

[10] J. Chen, V. Perebeinos, M. Freitag et al., "Applied physics: bright infrared emission from electrically induced excitons in carbon nanotubes," Science, vol. 310, no. 5751, pp. 1171-1174, 2005.

[11] J.-P. Salvetat, J.-M. Bonard, N. B. Thomson et al., "Mechanical properties of carbon nanotubes," Applied Physics A, vol. 69, no. 3, pp. 255-260, 1999.
[12] D. Jariwala, V. K. Sangwan, L. J. Lauhon, T. J. Marks, and M. C. Hersam, "Carbon nanomaterials for electronics, optoelectronics, photovoltaics, and sensing," Chemical Society Reviews, vol. 42, no. 7, pp. 2824-2860, 2013.

[13] V. Shanov, W. Cho, R. Malik et al., "CVD growth, characterization and applications of carbon nanostructured materials," Surface and Coatings Technology, vol. 230, pp. 77-86, 2013.

[14] M. S. Arnold, J. L. Blackburn, J. J. Crochet et al., "Recent developments in the photophysics of single-walled carbon nanotubes for their use as active and passive material elements in thin film photovoltaics," Physical Chemistry Chemical Physics, vol. 15, no. 36, pp. 14896-14918, 2013.

[15] S. Parveen, S. Rana, and R. Fangueiro, "A review on nanomaterial dispersion, microstructure, and mechanical properties of carbon nanotube and nanofiber reinforced cementitious composites," Journal of Nanomaterials, vol. 2013, Article ID 710175, 19 pages, 2013.

[16] J. Zhao and J. Zhu, "Electron microscopy and in situ testing of mechanical deformation of carbon nanotubes," Micron, vol. 42, no. 7, pp. 663-679, 2011.

[17] C. Wang, K. Takei, T. Takahashi, and A. Javey, "Carbon nanotube electronics-moving forward," Chemical Society Reviews, vol. 42, no. 7, pp. 2592-2609, 2013.

[18] Y. Wu, X. Lin, and M. Zhang, "Carbon nanotubes for thin film transistor: fabrication, properties, and applications," Journal of Nanomaterials, vol. 2013, Article ID 627215, 16 pages, 2013.

[19] M. Terrones, "Sharpening the chemical scissors to unzip carbon nanotubes: crystalline graphene nanoribbons," ACS Nano, vol. 4, no. 4, pp. 1775-1781, 2010.

[20] M. Terrones, A. R. Botello-Méndez, J. Campos-Delgado et al., "Graphene and graphite nanoribbons: morphology, properties, synthesis, defects and applications," Nano Today, vol. 5, no. 4, pp. 351-372, 2010.

[21] S. Dutta and S. K. Pati, "Novel properties of graphene nanoribbons: a review," Journal of Materials Chemistry, vol. 20, no. 38, pp. 8207-8223, 2010.

[22] Y. Zhu, S. Murali, W. Cai et al., "Graphene and graphene oxide: synthesis, properties, and applications," Advanced Materials, vol. 22, no. 35, pp. 3906-3924, 2010.

[23] Y. H. Wu, T. Yu, and Z. X. Shen, "Two-dimensional carbon nanostructures: fundamental properties, synthesis, characterization, and potential applications," Journal of Applied Physics, vol. 108, no. 7, Article ID 071301, 2010.

[24] J. C. Meyer, A. K. Geim, M. I. Katsnelson, K. S. Novoselov, T. J. Booth, and S. Roth, "The structure of suspended graphene sheets," Nature, vol. 446, no. 7131, pp. 60-63, 2007.

[25] U. Bangert, M. H. Gass, A. L. Bleloch, R. R. Nair, and A. K. Geim, "Manifestation of ripples in free-standing graphene in lattice images obtained in an aberration-corrected scanning transmission electron microscope," Physica Status Solidi A, vol. 206, no. 6, pp. 1117-1122, 2009.

[26] A. Fasolino, J. H. Los, and M. I. Katsnelson, "Intrinsic ripples in graphene," Nature Materials, vol. 6, no. 11, pp. 858-861, 2007.

[27] A. Grill, "Diamond-like carbon: state of the art," Diamond and Related Materials, vol. 8, no. 2-5, pp. 428-434, 1999.

[28] A. K. Bhaskar, V. N. Deshmukh, and L. Prajapati, "Carbon nanotube as a drug delivery system: a review," International Journal of Pharmacy and Technology, vol. 5, no. 2, pp. 2695-2711, 2013.

[29] M. Roldo and D. G. Fatouros, "Biomedical applications of carbon nanotubes," Annual Reports on the Progress of Chemistry C, vol. 109, pp. 10-35, 2013. 
[30] World Health Organization, Health Aspects of Air Pollution Results From the WHO Project "Systematic Review of Health Aspects of Air Pollution in Europe", WHO Regional Office for Europe, Copenhagen, Denmark, 2004.

[31] G. Oberdörster, E. Oberdörster, and J. Oberdörster, "Nanotoxicology: an emerging discipline evolving from studies of ultrafine particles," Environmental Health Perspectives, vol. 113, no. 7, pp. 823-839, 2005.

[32] C.-W. Lam, J. T. James, R. McCluskey, S. Arepalli, and R. L. Hunter, "A review of carbon nanotube toxicity and assessment of potential occupational and environmental health risks," Critical Reviews in Toxicology, vol. 36, no. 3, pp. 189-217, 2006.

[33] Department of Employment and Workplace Relations, Australian Safety and Compensation Council, "A review of the potential occupational health and safety implication of nanotechnology," Final Report, 2006.

[34] National Institute for Occupational Safety and Health, Approaches to Safe Nanotechnology: An Information exchange with NIOSH, Department of Health and Human Services, Centers for Disease Control and Prevention, 2007.

[35] C. L. Ursini, D. Cavallo, A. M. Fresegna et al., "Study of cytotoxic and genotoxic effects of hydroxyl-functionalized multiwalled carbon nanotubes on human pulmonary cells," Journal of Nanomaterials, vol. 2012, Article ID 815979, 9 pages, 2012.

[36] Y.-Y. Guo, J. Zhang, Y.-F. Zheng, J. Yang, and X.-Q. Zhu, "Cytotoxic and genotoxic effects of multi-wall carbon nanotubes on human umbilical vein endothelial cells in vitro," Mutation Research, vol. 721, no. 2, pp. 184-191, 2011.

[37] K. Yang and Z. Liu, "In vivo biodistribution, pharmacokinetics, and toxicology of carbon nanotubes," Current Drug Metabolism, vol. 13, no. 8, pp. 1057-1067, 2012.

[38] S. Sharifi, S. Behzadi, S. Laurent, M. Laird Forrest, P. Stroeve, and M. Mahmoudi, "Toxicity of nanomaterials," Chemical Society Reviews, vol. 41, no. 6, pp. 2323-2343, 2012.

[39] C. J. Humphreys, "Radiation effects," Ultramicroscopy, vol. 28, no. 1-4, pp. 357-358, 1989.

[40] R. F. Egerton, P. Li, and M. Malac, "Radiation damage in the TEM and SEM," Micron, vol. 35, no. 6, pp. 399-409, 2004.

[41] Z. Liu, Q. Zhang, and L.-C. Qin, "Accurate determination of atomic structure of multiwalled carbon nanotubes by nondestructive nanobeam electron diffraction," Applied Physics Letters, vol. 86, no. 19, Article ID 191903, pp. 1-3, 2005.

[42] A. Béché, J. L. Rouvière, J. P. Barnes, and D. Cooper, "Strain measurement at the nanoscale: comparison between convergent beam electron diffraction, nanobeam electron diffraction, high resolution imaging and dark field electron holography," Ultramicroscopy, vol. 131, pp. 10-23, 2013.

[43] J. M. Zuo, M. Gao, J. Tao, B. Q. Li, R. Twesten, and I. Petrov, "Coherent nano-area electron diffraction," Microscopy Research and Technique, vol. 64, no. 5-6, pp. 347-355, 2004.

[44] W. Robards and A. J. Wilson, Procedures in Electron Microscopy, John Wiley \& Sons, New York, NY, USA, 1998.

[45] D. B. Williams and C. B. Carter, Transmission Electron Microscopy: A Textbook For Materials Science, Springer, New York, NY, USA, 1996.

[46] R. F. Egerton, "Electron energy-loss spectroscopy in the TEM," Reports on Progress in Physics, vol. 72, no. 1, Article ID 016502, 2009.

[47] R. F. Egerton, Electron Energy Loss Spectroscopy in the Electron Microscope, Plenum Press, New York, NY, USA, 1996.
[48] R. F. Egerton, "Formulae for light-element microanalysis by electron energy-loss spectrometry," Ultramicroscopy, vol. 3, no. 2, pp. 243-251, 1978.

[49] R. F. Egerton, "K-shell ionization cross-sections for use in microanalysis," Ultramicroscopy, vol. 4, no. 2, pp. 169-179, 1979.

[50] R. D. Leapman, P. Rez, and D. F. Mayers, "K, L, and M shell generalized oscillator strengths and ionization cross sections for fast electron collisions," The Journal of Chemical Physics, vol. 72, no. 2, pp. 1232-1243, 1980.

[51] D. R. Liu and L. M. Brown, "Influence of some practical factors on background extrapolation in EELS quantification," Journal of Microscopy, vol. 147, no. 1, pp. 37-49, 1987.

[52] C. Colliex, "Electron energy loss in the electron microscope," in Advances in Optical and Electron Microscopy, R. Barer and V. E. Cosslet, Eds., vol. 9, Academic Press, New York, NY, USA, 1984.

[53] P. Castrucci, F. Tombolini, M. Scarselli et al., "Anharmonicity in single-wall carbon nanotubes as evidenced by means of extended energy loss fine structure spectroscopy analysis," Physical Review B, vol. 75, no. 3, Article ID 035420, 2007.

[54] J. J. Ritsko, N. O. Lipari, P. C. Gibbons, and S. E. Schnatterly, "Core excitons in tetrathiafulvalene-tetracyanoquinodimethane (TTF-TCNQ)," Physical Review Letters, vol. 37, no. 16, pp. 1068-1071, 1976.

[55] M. de Crescenzi, F. Tombolini, M. Scarselli et al., "Visible and near ultraviolet photocurrent generation in carbon nanotubes," Surface Science, vol. 601, no. 13, pp. 2810-2813, 2007.

[56] M. Scarselli, C. Scilletta, F. Tombolini et al., "Photon harvesting with multi wall carbon nanotubes," Superlattices and Microstructures, vol. 46, no. 1-2, pp. 340-346, 2009.

[57] M. Scarselli, C. Scilletta, F. Tombolini et al., "Multiwall carbon nanotubes decorated with copper nanoparticles: effect on the photocurrent response," Journal of Physical Chemistry C, vol. 113, no. 14, pp. 5860-5864, 2009.

[58] B. K. Teo and D. C. Joy, EXAFS Spectroscopy, Techniques, and Applications, Plenum Press, New York, NY, USA, 1981.

[59] B. K. Teo, EXAFS: Basic Principles and Data Analysis, vol. 9 of Inorganic Chemistry Concepts, Springer, Heidelberg, Germany, 1986.

[60] M. Diociaiuti, L. Lozzi, M. Passacantando, S. Santucci, P. Picozzi, and M. de Crescenzi, "Influence of non-dipolar terms on the $\mathrm{Cu} \mathrm{L} \mathrm{L}_{2,3}$ and $\mathrm{M}_{2,3}$ electron energy loss fine structure (EELFS) spectra in transmission and reflection mode," Journal of Electron Spectroscopy and Related Phenomena, vol. 82, no. 1-2, pp. 1-12, 1996.

[61] P. Castrucci, F. Tombolini, M. Scarselli et al., "Comparison of the local order in highly oriented pyrolitic graphite and bundles of single-wall carbon nanotubes by nanoscale extended energy loss spectra," Journal of Physical Chemistry C, vol. 113, no. 12, pp. 4848-4855, 2009.

[62] P. Castrucci, M. Scarselli, M. de Crescenzi et al., "Packinginduced electronic structure changes in bundled single-wall carbon nanotubes," Applied Physics Letters, vol. 87, no. 10, Article ID 103106, 2005.

[63] J. M. Zuo, I. Vartanyants, M. Gao, R. Zhang, and L. A. Nagahara, "Atomic resolution imaging of a carbon nanotube from diffraction intensities," Science, vol. 300, no. 5624, pp. 1419-1421, 2003.

[64] L. Persichetti, F. Tombolini, S. Casciardi et al., "Folding and stacking defects of graphene flakes probed by electron nanobeam," Applied Physics Letters, vol. 99, no. 4, Article ID 041904, 2011. 
[65] T. Eberlein, U. Bangert, R. R. Nair et al., "Plasmon spectroscopy of free-standing graphene films," Physical Review B, vol. 77, no. 23, Article ID 233406, 2008. 

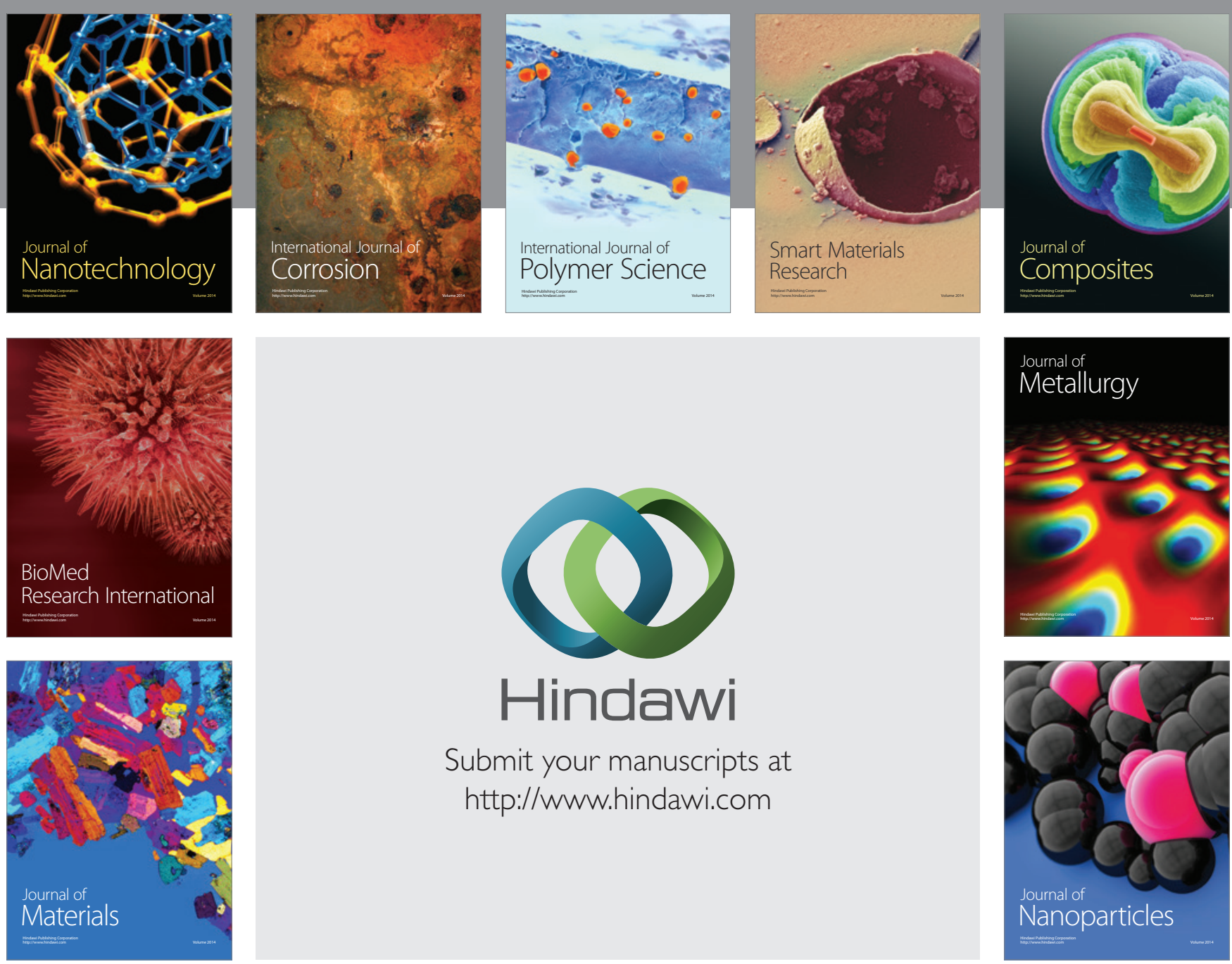

Submit your manuscripts at http://www.hindawi.com
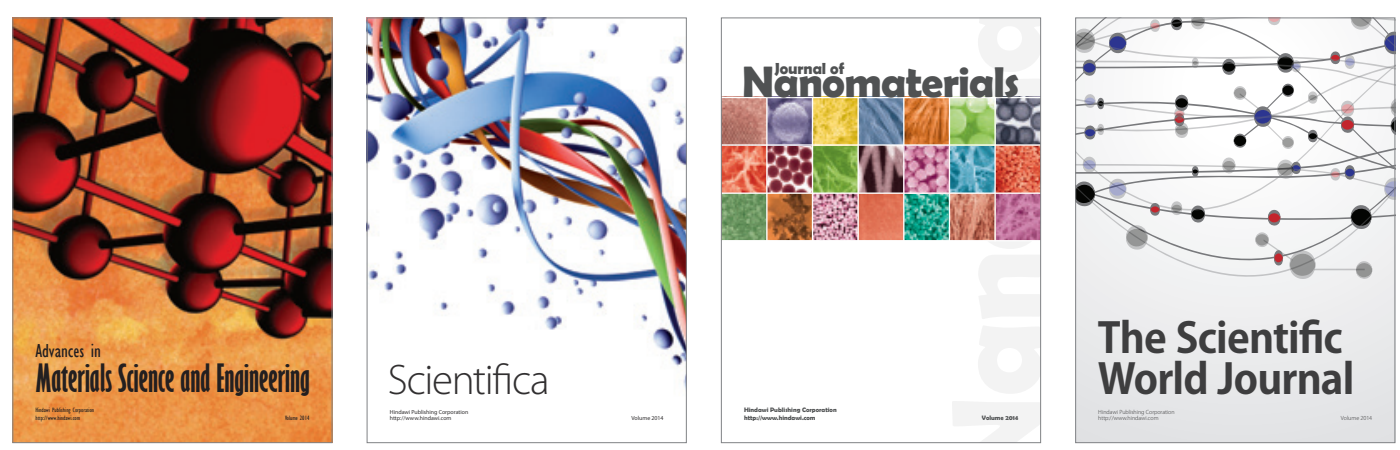

\section{The Scientific World Journal}
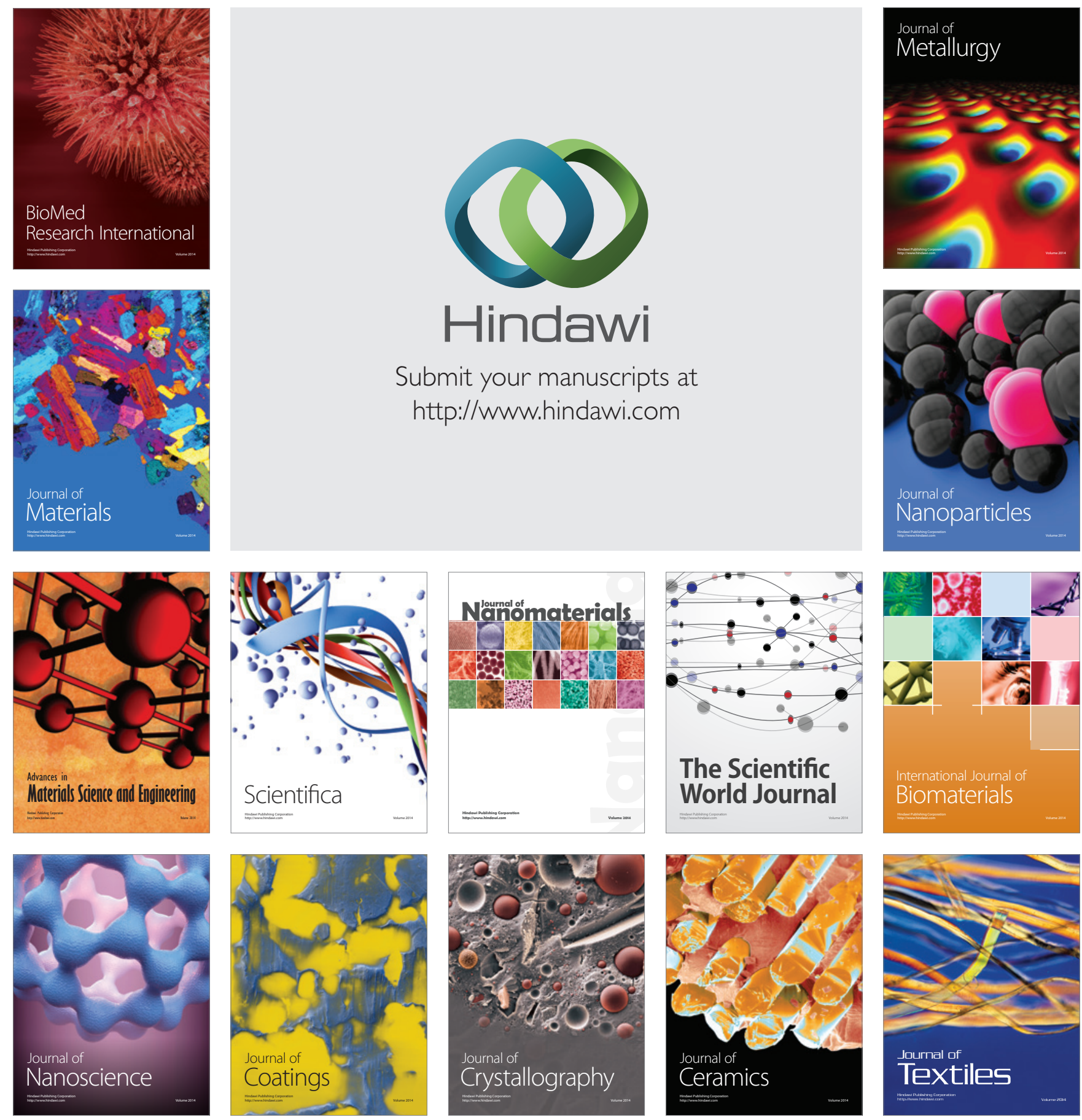